\title{
Just Say No: The Case Against Opioid-Based Postoperative Pain Management Regimens Following Breast Surgery
}

\author{
Theresa Schwartz \\ Breast Surgical Oncology, Henry Ford Health System, Detroit
}

Despite losing coverage in the headlines and priority among legislators due to the COVID-19 global health pandemic, the opioid crisis continues to wreak havoc across the country. Nearly 71,000 drug overdose deaths were reported by the Centers for Disease Control (CDC) in 2019 , with over 50,000 of these deaths involving opioids. ${ }^{1}$ It can trace its roots to the early 1990s when financial, ethical, and regulatory pressures, as well as false reassurances from pharmaceutical companies regarding the addictive nature of opioids, resulted in the encouragement of liberal use of opioids to control pain. ${ }^{2}$ The opioid epidemic blossomed due to altruistic efforts by physicians and surgeons attempting to provide compassionate patient care. The increased use of opioids subsequently resulted in widespread diversion and misuse, and current data suggest that $80 \%$ of people who use heroin first misused prescription opioids. ${ }^{3}$ This trend is both deadly and expensive. The CDC estimates that the total economic burden of prescription opioid misuse alone in the USA is US\$78.5 billion per year, including the costs of healthcare, lost productivity, addiction treatment, and criminal justice involvement. ${ }^{4}$

For many patients addicted to opioids, filling a prescription after an operation was where it all began. Approximately $6 \%$ of previously opioid-naïve patients continue to use opioids more than 3 months after an operation. ${ }^{5}$ Without data to guide management of acute postoperative pain, most patients are prescribed more

(C) Society of Surgical Oncology 2021

First Received: 22 March 2021

Accepted: 25 March 2021;

Published Online: 3 April 2021

T. Schwartz

e-mail: tschwar2@hfhs.org narcotics than necessary. In a systematic review of 810 postoperative patients in $2017,67-92 \%$ reported unused opioids. ${ }^{6}$ The vast majority (around $75 \%$ ) of these patients stored the extra opioids in unlocked locations, and less than $10 \%$ were discarded according to an appropriate disposal method, offering unlimited opportunities for diversion. Since the opioid epidemic was declared a public health emergency in 2017, priority has been placed on developing evidence-based strategies to both adequately treat postoperative acute pain and mitigate the consequences and side effects of the treatment provided.

In this issue of Annals of Surgical Oncology, Morin et al. ${ }^{7}$ add to the growing body of literature supporting nonopioid-based regimens for postoperative pain management following breast surgery. In their nonrandomized study, postoperative pain scores were compared amongst two cohorts of patients undergoing partial mastectomies-those who received an opioid-sparing regimen as part of a prospective pilot study (operation performed September 2017 to April 2019) and historical patients who were discharged with an opioid-based regimen (operation performed July 2015 to June 2016). The opioid-sparing regimen included preoperative education, gabapentin and acetaminophen given in holding, intraoperative liposomal bupivacaine and ketorolac, as well as postoperative acetaminophen and ibuprofen. They excluded patients with history of opioid dependence or chronic use as well as patients undergoing mastectomy or axillary surgery alone. Patients in the opioid-sparing group reported statistically significantly less pain on postoperative day 1 than patients in the traditional opioid group: $19.1 \%$ versus $34.3 \%$ reported moderate pain $(p<0.001)$, and $6.9 \%$ versus $15.7 \%$ reported severe pain $(p=0.004)$. By postoperative day 7 , the opioid-sparing group still reported less pain, but it did not reach statistical significance. The authors 
conclude that an opioid-sparing protocol following partial mastectomy is feasible, given its superior postoperative pain control compared with an opioid-based regimen.

Several prior studies confirm these findings. Kang et al. ${ }^{8}$ developed an opioid-sparing pathway for patients undergoing breast-conserving surgery with preoperative acetaminophen, pre-incision bupivacaine infiltration, postexcision bupivacaine wound deposition, intraoperative ketorolac, and postoperative acetaminophen and ibuprofen. Of the 226 patients treated with this regimen, $99 \%$ reported adequate pain control without opioids. Moo et al. ${ }^{9}$ similarly found successful pain control after breast-conserving surgery without narcotics. This group used intraoperative acetaminophen and ketorolac as well as operative site infiltration of bupivacaine and a discharge regimen of diclofenac and acetaminophen. Postoperative pain scores were statistically similar to their control group receiving opioids at discharge, suggesting no clinical necessity for opioids. Rojas et al. ${ }^{10}$ outlined a pilot Enhanced Recovery After Surgery (ERAS) protocol with many of the standard elements seen in other ERAS protocols. This group included preoperative counseling, acetaminophen, and gabapentin in holding, liposomal bupivacaine prior to incision as well as at time of closure, intraoperative ketorolac, and a discharge regimen of ibuprofen and acetaminophen. Comparing the pilot group with one receiving opioids, they found no statistically significant difference in postoperative pain scores.

We have the data to support adequate pain control without opioids following breast surgery. So why does it seem so difficult to change prescribing habits? The importance of one piece of ERAS protocols that makes them successful cannot be underestimated-preoperative counseling on postoperative expectations. Most patients are afraid of having pain and can be hesitant about a regimen that only includes over-the-counter medications. They may assume that it will not be enough and want an opioid prescription "just in case." Surgeons may fear being perceived as uncaring if they do not prescribe opioids. All fears, assumptions, and perceptions can be alleviated by a conversation with our patients prior to operation. Providing educational materials on the dangers of opioid use and the deadly effects of diversion, as well as a description of the pain regimen being prescribed, would give confidence to patients regarding the benefits of an opioid-sparing protocol. This is not an easy request, as all surgeons are being asked to do more in less time, but our prescribing habits have a tremendous impact on the opioid epidemic. We cannot just watch from the sidelines.

A one-paragraph letter to the editor written in the New England Journal of Medicine (NEJM) in 1980 titled "Addiction Rare in Patients Treated with Narcotics" reported only four opioid addictions in nearly 12,000 patients who received at least one opioid while hospitalized. Despite only including patients being treated in a controlled in-patient setting with medications delivered as prescribed, with no data provided, this 101-word letter has been cited 608 times. In 2017, a correspondence was written in the $N E J M^{11}$ outlining the unintended effects of this letter-and noted that $72 \%$ of the authors who cited it did so as evidence that addiction was rare in patients treated with opioids, over $80 \%$ of the authors who cited it did not note that the patients they reference in the letter were hospitalized at the time of opioid administration, and other authors grossly misrepresented the conclusions. Why was it so easy to believe five sentences of commentary, yet so difficult to trust the numerous well-designed studies showing how opioid-sparing regimens provide adequate postoperative pain control? Opioids simply are not necessary. We have the data. It is time for all surgeons to start using it.

\section{REFERENCES}

1. Ahmad FB, Rossen LM, Sutton P. Provisional drug overdose death counts. National Center for Health Statistics. 2021.

2. Baker DW. History of the Joint Commission's pain standards: lessons for today's prescription opioid epidemic. JAMA. 2017;317(11):1117-8.

3. Muhuri PK, Gfroerer JC, Davies MC. Associations of nonmedical pain reliever use and initiation of heroin use in the United States. CBHSQ Data Rev. 2013.

4. Florence CS, Zhou C, Luo F, Xu L. The economic burden of prescription opioid overdose, abuse, and dependence in the United States, 2013. Med Care. 2016;54(10):901-6.

5. Brummett CM, Waljee JF, Goesling J, et al. New persistent opioid use after minor and major surgical procedures in US adults. JAMA Surg. 2017;152(6):e170504-e170504.

6. Bicket MC, Long JJ, Pronovost PJ, Alexander GC, Wu CL. Prescription opioid analgesics commonly unused after surgery: a systematic review. JAMA Surg. 2017;152(11):1066-71.

7. Morin $\mathrm{CM}$ et al. Opioid-sparing multimodal analgesia protocol for lumpectomy patients results in superior postoperative pain control. Ann Surg Oncol. 2021. https://doi.org/10.1245/s10434021-09990-0.

8. Kang R, Read JT, Glaser AC, Barth RJ. Eliminating opioids from breast conserving surgery: perioperative pain management pathway. J Am Coll Surg. 2020;230:975-82.

9. Moo TA, Assel M, Yeahia R, Nierstedt R, Van Zee KJ, Kirstein LJ, Vickers A, Morrow M, Twersky R. Routine opioid prescriptions are not necessary after breast excisional biopsy or lumpectomy procedures. Ann Surg Oncol. 2021;28:303-9.

10. Rojas K, Manasseh DM, Flom PL, Agbroko A, Bilbro N, Andaz C, Borgen PI. A pilot study of breast surgery Enhanced Recovery After Surgery (ERAS) protocol to eliminate narcotic prescription at discharge. Breast Cancer Res Treat. 2018;171(3):621-6.

11. Leung P, Macdonald EM, Dhalla IA, Juurlink DN. A 1980 letter on the risk of opioid addiction. $N$ Engl J Med. 2017;376:2194-5.

Publisher's Note Springer Nature remains neutral with regard to jurisdictional claims in published maps and institutional affiliations. 